TRANSACTIONS OF THE

AMERICAN MATHEMATICAL SOCIETY

Volume 358, Number 9, September 2006, Pages 3969-3983

S 0002-9947(06)04017-7

Article electronically published on April 11, 2006

\title{
TOROIDAL ORBIFOLDS, GERBES AND GROUP COHOMOLOGY
}

\author{
ALEJANDRO ADEM AND JIANZHONG PAN
}

\begin{abstract}
In this paper we compute the integral cohomology of certain semidirect products of the form $\mathbb{Z}^{n} \rtimes G$, arising from a linear $G$ action on the $n$-torus, where $G$ is a finite group. The main application is the complete calculation of torsion gerbes for six-dimensional examples arising in string theory.
\end{abstract}

\section{INTRODUCTION}

During the last several years, there has been a surge of interest in the mathematics involved in studying various twisting processes in string theory. One of the earliest ones was the notion of a $B$-field on a smooth manifold. A $B$-field can be interpreted topologically as a class in $H^{3}(X, \mathbb{Z})$. In particular the torsion classes, or the so-called flat B-fields, appear to be geometrically significant. Another source of motivation comes from stringy orbifold theory. The twisting process on orbifolds is much more subtle due to the presence of a local finite group action. In the case of a global quotient orbifold $X / G$, the first notion of twisting - discrete torsion - was proposed by Vafa [12: a discrete torsion is a cohomology class $\alpha \in H^{2}(G, U(1))$, where $G$ is a finite group. It has also been suggested that discrete torsion relates to desingularization. Recall that there are two methods to remove singularities, resolution and deformation. Both play important roles in the theory of CalabiYau 3-folds. A smooth manifold $Y$ obtained from an orbifold $X$ via a sequence of resolutions and deformations is called a desingularization of $X$. In string theory, we also require the resolutions to be crepant resolutions. It is known that such a desingularization may not exist in dimensions higher than three. In this case, we allow our desingularization to be an orbifold. Vafa and Witten [11] proposed discrete torsion as a parameter for deformation. However, this proposal immediately runs into trouble because the number of desingularizations is much larger than the number of discrete torsion elements. For example, D. Joyce 8 constructed five different desingularizations of an orbifold of the form $\mathbb{T}^{6} / \mathbb{Z} / 4$ (see Example 1.2 below), while $H^{2}(\mathbb{Z} / 4, U(1))=0$. To count these "missing" desingularizations, Ruan 10. proposed the notion of an inner local system. In the framework of gerbes, an inner local system can be interpreted as a holonomy line bundle (see [9]).

Received by the editors June 10, 2004.

2000 Mathematics Subject Classification. Primary 20 J06.

Key words and phrases. Orbifolds, gerbes, group cohomology.

The first author was partially supported by the NSF, and the second author was partially supported by NSFC project 19701032. 
The computation of gerbes can be a difficult problem. For the examples arising from orbifolds, almost all the known computations are from discrete torsion. A priori we do not even know if gerbes capture Joyce's example, which is what we would like to hope for. It seems then that to understand gerbes better it will be necessary to compute them for physically significant examples. This is the main motivation for our paper.

Here we establish a cohomology computation for groups arising as orbifold fundamental groups associated to examples from geometry and string theory. In particular our computations apply to six-dimensional examples arising from finite group actions on tori, and we obtain some rather interesting examples of torsion gerbes, which should have physical significance. From the point of view of group cohomology, we are extending previous work (see [1]), where a number of calculations for equivariant cohomology were explicitly obtained. Our mathematical interest was rekindled by more recent contributions related to physics, such as the work appearing in [3], where a comprehensive description of computations for four-dimensional examples was provided.

We now outline the basic mathematical framework for our paper. Let $G \subset$ $G L_{n}(\mathbb{Z})$ denote a finite subgroup; then it will act linearly on $\mathbb{R}^{n}$ preserving a lattice $\mathbb{Z}^{n} \subset \mathbb{R}^{n}$. Hence there is an induced linear action on the torus $\mathbb{T}^{n}$. Our basic goal will be to compute the equivariant cohomology $H^{*}\left(E G \times_{G} \mathbb{T}^{n}, \mathbb{Z}\right)$. Now the Borel construction $E G \times_{G} \mathbb{T}^{n}$ will be aspherical, whence it is an EilenbergMac Lane space of type $K\left(\pi_{1}\left(E G \times_{G} \mathbb{T}^{n}\right), 1\right)$. If we let $\Gamma=\pi_{1}\left(E G \times_{G} \mathbb{T}^{n}\right)$, then the problem we are raising is that of computing the low-dimensional cohomology of the discrete group $\Gamma$. Note that $\Gamma$ can also be identified with the orbifold fundamental group of $\mathbb{T}^{n} \rightarrow \mathbb{T}^{n} / G$. As a consequence of our calculations, we will be able to compute the group of isomorphism classes of (flat) gerbes for these orbifolds, as these invariants can be identified with the (second) third equivariant cohomology group of the manifold with $(U(1))$ integral coefficients. In other words the invariants we will be interested in computing are $F G b\left(\mathbb{T}^{n} / G\right)=H^{2}\left(E G \times_{G} \mathbb{T}^{n}, U(1)\right)$ and $G b\left(\mathbb{T}^{n} / G\right)=H^{3}\left(E G \times{ }_{G} \mathbb{T}^{n}, \mathbb{Z}\right)$.

For all of these actions, $[0] \in \mathbb{R}^{n} / \mathbb{Z}^{n}$ is a fixed point. This implies that there is a geometric section for $E G \times_{G} \mathbb{T}^{n}$, hence also an algebraic section for $\Gamma \rightarrow$ $G$. Therefore $\Gamma \cong \pi_{1}\left(\mathbb{T}^{n}\right) \rtimes G=\mathbb{Z}^{n} \rtimes G$, a semi-direct product. An important ingredient here will be the detailed structure of the $\mathbb{Z} G$-lattice $\mathbb{Z}^{n} \mathbb{1}$ Indeed, the semi-direct product is completely determined by this $\mathbb{Z}[G]$-module structure.

The following theorem is the basic computational device which we will use:

Theorem 1.1. If $M$ is a $\mathbb{Z} G$-lattice which restricts on each $p$-Sylow subgroup to a direct sum of finitely many one- and two-dimensional integral representations, then for all integers $k \geq 0$, we have

$$
H^{k}(M \rtimes G, \mathbb{Z}) \cong \bigoplus_{i+j=k} H^{i}\left(G, H^{j}(M, \mathbb{Z})\right) .
$$

Having established this, we turn our attention to examples of interest in physics. Computations for four-dimensional models were explicitly described in [3]. However, for all of those examples, the odd-dimensional cohomology vanishes, hence no interesting torsion gerbes can appear. In contrast, the six-dimensional examples

\footnotetext{
${ }^{1}$ If $G$ is a finite group, a $\mathbb{Z} G$-lattice is a $\mathbb{Z} G$-module which happens to be a free abelian group
} of finite rank. 
are far more interesting and much harder to compute. In particular we consider the following important examples studied in [8] and [1].

Example 1.2. Let $Y_{1}=T^{6} / \mathbb{Z} / 4$, where the action is induced from that on $\mathbb{C}^{3}$, given by

$$
\tau\left(z_{1}, z_{2}, z_{3}\right)=\left(-z_{1}, i z_{2}, i z_{3}\right) .
$$

Example 1.3. Let $Y_{2}=T^{6} / \mathbb{Z} / 2 \times \mathbb{Z} / 2$, where the action is induced from that on $\mathbb{C}^{3}$, given by

$$
\tau_{1}\left(z_{1}, z_{2}, z_{3}\right)=\left(-z_{1},-z_{2}, z_{3}\right), \tau_{2}\left(z_{1}, z_{2}, z_{3}\right)=\left(-z_{1}, z_{2},-z_{3}\right) .
$$

Our techniques yield the following complete calculations for the corresponding groups of gerbes:

$$
\begin{gathered}
F G b\left(Y_{1}\right)=U(1)^{5} \oplus(\mathbb{Z} / 2)^{4}, G b\left(Y_{1}\right)=\mathbb{Z}^{4} \oplus(\mathbb{Z} / 2)^{4} \\
F G b\left(Y_{2}\right)=U(1)^{3} \oplus(\mathbb{Z} / 2)^{18}, G b\left(Y_{2}\right)=\mathbb{Z}^{8} \oplus(\mathbb{Z} / 2)^{18} .
\end{gathered}
$$

Note the rather mysterious torsion subgroup $(\mathbb{Z} / 2)^{4}$ for $Y_{1}$ and $(\mathbb{Z} / 2)^{18}$ for $Y_{2}$. We expect that it will have physical significance, and it would be interesting to build a geometric model for these torsion gerbes. Their role in twisted orbifold invariants (see [10]) also deserves further study. However, we should point out that a consequence of our computations is the conclusion that it is too naive to expect that gerbes can capture the different desingularizations of an orbifold. Instead we see that cohomology calculations for the classifying space of an orbifold supply intricate invariants which will measure the complicated nature of the possible resolutions, but are far from determining them.

The methods used in this paper are from the cohomology of groups. Applying a construction from 4 we will manufacture a very simple projective resolution for certain semidirect products of the form $\mathbb{Z}^{n} \rtimes G$, where $G$ is a finite group. The key point will be that the resolution will allow us to prove - at the cochain level - the collapse (at the $E_{2}$ stage) of the Lyndon-Hochschild-Serre spectral sequence for the group extension. The methods are actually very similar to those used to compute the cohomology of wreath products (Nakaoka's Theorem; see [7, page 50).

We should mention that computing the cohomology of groups such as these (including crystallographic groups) is an important problem in the cohomology of groups. More specifically, the exact determination of the Schur multiplier $H^{2}\left(\mathbb{Z}^{n} \rtimes G, U(1)\right)$ seems like an important basic question.

The authors would like to thank D. Benson, A. Klemm and Y. Ruan for their comments and advice.

\section{Cohomology of Semidirect Products}

General references for group cohomology are the books by Adem-Milgram [2], Brown [5] and Evens [7]. We will assume a basic familiarity with the material on group extensions and their cohomology.

The key input which we will use is a result due to Brady [4, which will allow us to construct an extremely practical projective resolution and from there compute the cohomology of the groups we will be interested in. The basic approach will be 
the following: given certain conditions on the $\mathbb{Z} G$-lattice $M$, the cohomology of the semidirect product $M \rtimes G$ can be completely computed 2

Let $\Gamma=M \rtimes G$, where $G$ acts on the group $M$ by a homomorphism $\phi: G \rightarrow$ Aut $(M)$. We write $m^{g}$ for $\phi(g) m$, where $m \in M, g \in G$.

Definition 2.1. Given a free resolution $\epsilon: F \rightarrow \mathbb{Z}$ of $\mathbb{Z}$ over $\mathbb{Z}[M]$, we say that it admits an action of $G$ compatible with $\phi$ if for all $g \in G$ there is an augmentationpreserving chain map $\tau(g): F \rightarrow F$ such that

(1) $\tau(g)[m \cdot f]=m^{g} \cdot[\tau(g) f]$ for all $m \in M$ and $f \in F$,

(2) $\tau(g) \tau\left(g^{\prime}\right)=\tau\left(g g^{\prime}\right)$ for all $g, g^{\prime} \in G$,

(3) $\tau(1)=1_{F}$.

If such an action exists we can give $F$ a $\Gamma$-module structure as follows. If $\gamma \in \Gamma$, then $\gamma$ can be expressed uniquely as $\gamma=m g$, with $m \in M$ and $g \in G$. We set $\gamma \cdot f=(m g) \cdot f=m \cdot \tau(g) f$. Note that given any $G$-module $L$, this inflates to a $\Gamma$-action on $L$ via the projection $\Gamma \rightarrow G$.

Proposition 2.2. If $\epsilon: F \rightarrow \mathbb{Z}$ is a free resolution of $\mathbb{Z}$ over $\mathbb{Z}[M]$, which admits an action of $G$ compatible with $\phi$, and $\epsilon^{\prime}: P \rightarrow \mathbb{Z}$ is any free resolution of $\mathbb{Z}$ over $G$, then $\epsilon^{\prime} \otimes \epsilon: P \otimes F \rightarrow \mathbb{Z}$ is a free resolution of $\mathbb{Z}$ over $\mathbb{Z} \Gamma$.

Proof. It suffices to show that each chain module is $\mathbb{Z} \Gamma$-free. Indeed, if $F_{i}$ restricts on $M$ to a free module of rank $n_{i}$, then we have

$$
\mathbb{Z} G \otimes F_{i} \cong \mathbb{Z}[\Gamma / M] \otimes F_{i} \cong \mathbb{Z} \Gamma \otimes_{M}(\mathbb{Z}[M])^{n_{i}} \cong(\mathbb{Z} \Gamma)^{n_{i}}
$$

In the case $\Gamma=\mathbb{Z}^{n} \rtimes G$, a particular free resolution for $\mathbb{Z}^{n}$ can be chosen as follows. Let $\partial: \mathbb{Z}[\mathbb{Z}] \rightarrow \mathbb{Z}[\mathbb{Z}]$ be given by $\partial(u)=(1-x) u$, where $x$ is a generator for the infinite cyclic group. Then one can verify that $\partial$ is injective, with cokernel the trivial module $\mathbb{Z}$. Denote this free resolution by $D$; take $n$ copies of it, say denoted by $D_{1}, D_{2}, \ldots, D_{n}$. Now let $F=D_{1} \otimes D_{2} \otimes \cdots \otimes D_{n}$; this will be a free resolution for the trivial module $\mathbb{Z}$ over the group ring $\mathbb{Z}\left[\mathbb{Z}^{n}\right]$. We observe the following: $\operatorname{Hom}_{\mathbb{Z}[\mathbb{Z}]}(D, \mathbb{Z})$ has trivial differentials. Using the Kunneth formula, we infer that the analogous fact will hold for the resolution $F$. Hence we have that the cochain complex for computing the cohomology $H^{*}\left(\mathbb{Z}^{n}, \mathbb{Z}\right)$ is in fact additively isomorphic to the cohomology. We shall call this a special resolution.

Now suppose we are given $\Gamma=\mathbb{Z}^{n} \rtimes G$ and the existence of a compatible action of $G$ on this special resolution $F$ for $\mathbb{Z}^{n}$. Then we can apply the ideas 3 above as follows.

Theorem 2.3. Let $\epsilon: F \rightarrow \mathbb{Z}$ be a special free resolution of $\mathbb{Z}$ over $\mathbb{Z}^{n}$, and suppose that there is a compatible action of $G$ on $F$. Then for all integers $k \geq 0$, we have

$$
H^{k}\left(\mathbb{Z}^{n} \rtimes G, \mathbb{Z}\right)=\bigoplus_{i+j=k} H^{i}\left(G, H^{j}\left(\mathbb{Z}^{n}, \mathbb{Z}\right)\right) .
$$

\footnotetext{
${ }^{2}$ Recall that given a split extension with a prescribed action on an abelian kernel, the resulting group is uniquely determined up to isomorphism.

${ }^{3}$ Our proof is very similar to Evens' proof of Nakaoka's Theorem on the cohomology of wreath products (see 7], pages 19 and 50). We are grateful to Dave Benson for this observation.
} 
Proof. Let $\epsilon^{\prime} \otimes \epsilon: P \otimes F \rightarrow \mathbb{Z}$ be the free resolution of $\mathbb{Z}$ over $\Gamma$ which is given by Proposition 2.2. Then the cohomology of $\Gamma$ can be computed as the cohomology of $\operatorname{Hom}_{\Gamma}(P \otimes F, \mathbb{Z})$. Note however that the normal subgroup $\Gamma^{\prime}=$ $\mathbb{Z}^{n}$ acts trivially on $P$, whence we obtain the isomorphism $H_{\Gamma}(P \otimes F, \mathbb{Z}) \cong$ $\operatorname{Hom}_{\Gamma / \Gamma^{\prime}}\left(P, \operatorname{Hom}_{\Gamma^{\prime}}(F, \mathbb{Z})\right)$. Hence our computation has reduced to calculating the $G$ equivariant cohomology of a $\mathbb{Z} G$-cochain complex with trivial differentials, from which the result immediately follows.

To conclude the section, we will state three simple but useful lemmas which facilitate the construction of compatible actions; their proofs are straightforward.

Lemma 2.4. If $\epsilon_{i}: F_{i} \rightarrow \mathbb{Z}$ is a projective $M_{i}$-resolution of $\mathbb{Z}$ for $i=1,2$, then $\epsilon_{1} \otimes \epsilon_{2}: F_{1} \otimes F_{2} \rightarrow \mathbb{Z}$ is a projective $M$-resolution of $\mathbb{Z}$, where $M=M_{1} \times M_{2}$. If, furthermore, a group $G$ acts compatibly on $F_{i}$ by $\tau_{i}$ for $i=1,2$, then a compatible action of $G$ on $\epsilon_{1} \otimes \epsilon_{2}: F_{1} \otimes F_{2} \rightarrow \mathbb{Z}$ is given by $\tau(g)\left(f_{1} \otimes f_{2}\right)=\tau_{1}(g)\left(f_{1}\right) \otimes \tau_{2}(g)\left(f_{2}\right)$.

Lemma 2.5. If $G=G_{1} \times G_{2}, M$ is a $G_{i}$-module for $i=1,2$, and $G_{i}$ acts compatibly by $\tau_{i}$ on a $M$-resolution of $\mathbb{Z}, \epsilon: F \rightarrow \mathbb{Z}$ such that $\tau_{1}\left(g_{1}\right) \tau_{2}\left(g_{2}\right)=\tau_{2}\left(g_{2}\right) \tau_{1}\left(g_{1}\right)$, then a compatible action of $G$ on $\epsilon: F \rightarrow \mathbb{Z}$ is given by $\tau(g)(f)=\tau_{1}(g) \tau_{2}(g) f$.

Lemma 2.6. If $M$ is a $G_{1}$-module, $\pi: G_{2} \rightarrow G_{1}$ a group homomorphism, and $\epsilon: F \rightarrow \mathbb{Z}$ is a $M$-resolution of $\mathbb{Z}$ such that $G_{1}$ acts compatibly on it by $\tau^{\prime}$, then $G_{2}$ also acts compatibly on it via $\tau(g) f=\tau^{\prime}(\pi(g)) f$ for any $g \in G_{2}$.

\section{Constructing compatible actions}

Consider the special $\mathbb{Z}[\mathbb{Z}]$-resolution of $\mathbb{Z}$ :

$$
0 \rightarrow \mathbb{Z}[\mathbb{Z}] a_{1} \rightarrow \mathbb{Z}[\mathbb{Z}] a_{0} \rightarrow \mathbb{Z}
$$

with generators $a_{i}$ in degree $i$ for $i=0,1$. If we tensor it with another copy of the same resolution, but now with generators $b_{0}, b_{1}$, we obtain the explicit special resolution for the group $\mathbb{Z}^{2}=\mathbb{Z} x_{1} \oplus \mathbb{Z} x_{2}$ :

$$
\mathbb{Z}\left[\mathbb{Z}^{2}\right] a_{1} \otimes b_{1} \rightarrow \mathbb{Z}\left[\mathbb{Z}^{2}\right] a_{0} \otimes b_{1} \oplus \mathbb{Z}\left[\mathbb{Z}^{2}\right] a_{1} \otimes b_{0} \rightarrow \mathbb{Z}\left[\mathbb{Z}^{2}\right] a_{0} \otimes b_{0},
$$

where the boundary maps are given by

$$
\begin{gathered}
\partial\left(a_{1} \otimes b_{1}\right)=\left(1-x_{1}\right) a_{0} \otimes b_{1}-\left(1-x_{2}\right) a_{1} \otimes b_{0}, \\
\partial\left(a_{0} \otimes b_{1}\right)=\left(1-x_{2}\right) a_{0} \otimes b_{0}, \partial\left(a_{1} \otimes b_{0}\right)=\left(1-x_{1}\right) a_{0} \otimes b_{0} .
\end{gathered}
$$

In what follows we will be constructing compatible finite group actions on this explicit complex. The action on $\left\langle x_{1}, x_{2}\right\rangle$ is given as follows. If $A \in G L_{2}(\mathbb{Z})$, then we identify $x_{1}$ with $(1,0)$ and $x_{2}$ with $(0,1)$, respectively. Then the action is of the form

$$
(1,0) \mapsto(1,0) A=\left(a_{11}, a_{12}\right), \quad(0,1) \mapsto(0,1) A=\left(a_{21}, a_{22}\right)
$$

which multiplicatively becomes

$$
x_{1} \mapsto x_{1}^{a_{11}} x_{2}^{a_{12}}, \quad x_{2} \mapsto x_{1}^{a_{21}} x_{2}^{a_{22}} .
$$

Given a generator $t \in G$, we can identify it with a $2 \times 2$ matrix $A \in G L_{2}(\mathbb{Z})$ as above. We now describe an explicit procedure for constructing $\tau(t): F \rightarrow F$. First we observe that as we want it to be augmentation-preserving, we assume that the generator $a_{0} \otimes b_{0}$ is fixed. Next we set up the algebraic equations which must be solved to define $\tau(t)$ as a chain map. 
For brevity we write $\tau$ instead of $\tau(t)$. We have

$$
\tau\left(a_{0} \otimes b_{1}\right)=q_{01} a_{0} \otimes b_{1}+q_{10} a_{1} \otimes b_{0}, \quad \tau\left(a_{1} \otimes b_{0}\right)=r_{01} a_{0} \otimes b_{1}+r_{10} a_{1} \otimes b_{0}
$$

for elements $r_{i j}, q_{i j} \in \mathbb{Z}\left[\mathbb{Z}^{2}\right]$. Now the equation $\partial \tau=\tau \partial$ is equivalent to the following algebraic conditions on these coefficients:

$q_{01}\left(1-x_{2}\right)+q_{10}\left(1-x_{1}\right)=1-x_{1}^{a_{21}} x_{2}^{a_{22}}, \quad r_{01}\left(1-x_{2}\right)+r_{10}\left(1-x_{1}\right)=1-x_{1}^{a_{11}} x_{2}^{a_{12}}$.

Similarly let us assume that $\tau\left(a_{1} \otimes b_{1}\right)=q_{11} a_{1} \otimes b_{1}$. Then the chain complex condition implies the following equations:

$$
\begin{gathered}
q_{11}\left(1-x_{1}\right)=\left(1-x_{1}^{a_{11}} x_{2}^{a_{12}}\right) q_{01}-\left(1-x_{1}^{a_{21}} x_{2}^{a_{22}}\right) r_{01}, \\
-q_{11}\left(1-x_{2}\right)=\left(1-x_{1}^{a_{11}} x_{2}^{a_{12}}\right) q_{10}-\left(1-x_{1}^{a_{21}} x_{2}^{a_{22}}\right) r_{10} .
\end{gathered}
$$

Theorem 3.1. Let $M$ be a $\mathbb{Z} G$-lattice which is a direct sum of indecomposable $G$-submodules of $\mathbb{Z}$-rank at most two. Then there is a compatible action of $G$ on the special $M$-resolution of $\mathbb{Z}$ if $G$ is as follows:

(1) $G=\mathbb{Z} / n$ for $n=2,3,4$,

(2) $G=\mathbb{Z} / 2 \oplus \mathbb{Z} / 2$,

(3) $G=D_{8}$, the dihedral group of order 8 .

Proof. We will be considering isomorphism classes of $\mathbb{Z} G$-lattices. Note that by Lemma 2.4 it suffices to prove the theorem when the module is indecomposable of rank one or two. On the other hand, for rank one modules, it suffices to prove the theorem for $G=\mathbb{Z} / 2$ by Lemma 2.6. Now we proceed to prove the theorem case by case.

(1) $G=\mathbb{Z} / 2$ with generator $t$; the only nontrivial indecomposable $G$-modules of $\mathbb{Z}$-rank at most 2 are given by the matrix representing $t:(-1)$ and

$$
A=\left(\begin{array}{cc}
0 & 1 \\
1 & 0
\end{array}\right) .
$$

For the first case, $\tau(t)\left(a_{0}\right)=a_{0}$ and $\tau(t)\left(a_{1}\right)=-x^{-1} a_{1}$. It is easy to verify that this gives a compatible action of $\mathbb{Z} / 2$ on the above resolution.

For the second case, we use the special $\mathbb{Z}\left[\mathbb{Z}^{2}\right]$-resolution of $\mathbb{Z}$ described above, with free generators denoted by $a_{i} \otimes b_{j}$, where $i, j=0,1$. For this example, the equations above admit the following simple solutions: $q_{01}=0, q_{10}=1, r_{01}=1$, $r_{10}=0, q_{11}=-1$. Hence the following definition defines a chain map satisfying the first compatibility condition:

$$
\begin{gathered}
\tau(t)\left(a_{0} \otimes b_{0}\right)=a_{0} \otimes b_{0}, \quad \tau(t)\left(a_{1} \otimes b_{0}\right)=a_{0} \otimes b_{1}, \\
\tau(t)\left(a_{0} \otimes b_{1}\right)=a_{1} \otimes b_{0}, \quad \tau(t)\left(a_{1} \otimes b_{1}\right)=-a_{1} \otimes b_{1} .
\end{gathered}
$$

Moreover, in this case one can easily verify that this gives a compatible action of $\mathbb{Z} / 2$ on the above resolution, i.e., $\tau(t)^{2}=I d: F \rightarrow F$.

(2) $G=\mathbb{Z} / 3$ with generator $t$; the only non-trivial indecomposable $\mathbb{Z} G$-module of $\mathbb{Z}$-rank two can be represented by the matrix

$$
A=\left(\begin{array}{cc}
0 & -1 \\
1 & -1
\end{array}\right)
$$


In this case the solutions to the equations are incorporated into the following formula for $\tau$ :

$$
\begin{array}{llc}
\tau(t)\left(a_{0} \otimes b_{0}\right) & = & a_{0} \otimes b_{0}, \\
\tau(t)\left(a_{1} \otimes b_{0}\right) & = & -x_{2}^{-1} a_{0} \otimes b_{1}, \\
\tau(t)\left(a_{0} \otimes b_{1}\right) & = & a_{1} \otimes b_{0}-x_{1} x_{2}^{-1} a_{0} \otimes b_{1}, \\
\tau(t)\left(a_{1} \otimes b_{1}\right)= & x_{2}^{-1} a_{1} \otimes b_{1} .
\end{array}
$$

We will give a complete proof that this indeed gives a compatible action of $\mathbb{Z} / 3$ on the special resolution. We need to check condition (2) in Definition 2.1 which in this case is $\tau(t)^{3}=I d$ :

$$
\begin{aligned}
& \tau(t)^{3}\left(a_{1} \otimes b_{0}\right)=\quad \tau(t)^{2}\left(-x_{2}^{-1} a_{0} \otimes b_{1}\right) \\
& =\quad-\tau(t)\left(x_{2} x_{1}^{-1}\left(a_{1} \otimes b_{0}-x_{1} x_{2}^{-1} a_{0} \otimes b_{1}\right)\right) \\
& =\quad \tau(t)\left(a_{0} \otimes b_{1}-x_{2} x_{1}^{-1} a_{1} \otimes b_{0}\right) \\
& =\quad a_{1} \otimes b_{0}-x_{1} x_{2}^{-1} a_{0} \otimes b_{1}+x_{1} x_{2}^{-1} a_{0} \otimes b_{1} \\
& =\quad a_{1} \otimes b_{0}, \\
& \tau(t)^{3}\left(a_{0} \otimes b_{1}\right)=\tau(t)^{2}\left(a_{1} \otimes b_{0}-x_{1} x_{2}^{-1} a_{0} \otimes b_{1}\right) \\
& =\tau(t)\left(-x_{2}^{-1} a_{0} \otimes b_{1}-x_{1}^{-1}\left(a_{1} \otimes b_{0}-x_{1} x_{2}^{-1} a_{0} \otimes b_{1}\right)\right) \\
& =\quad \tau(t)\left(-x_{1}^{-1} a_{1} \otimes b_{0}\right) \\
& =\quad-x_{2}\left(-x_{2}^{-1} a_{0} \otimes b_{1}\right)=a_{0} \otimes b_{1}, \\
& \tau(t)^{3}\left(a_{1} \otimes b_{1}\right)=\quad \tau^{2}\left(x_{2}^{-1} a_{1} \otimes b_{1}\right) \\
& =\quad \tau(t)\left(x_{2} x_{1}^{-1} x_{2}^{-1} a_{1} \otimes b_{1}\right) \\
& =\quad \tau(t)\left(x_{1}^{-1} a_{1} \otimes b_{1}\right) \\
& \left.=\quad x_{2} x_{2}^{-1} a_{1} \otimes b_{1}\right) \\
& =\quad a_{1} \otimes b_{1} \text {. }
\end{aligned}
$$

(3) $G=\mathbb{Z} / 4$ with generator $t$; the only faithful indecomposable $\mathbb{Z} / 4$-lattice of $\mathbb{Z}$-rank 2 is given by the matrix representing $t$ :

$$
A=\left(\begin{array}{ll}
0 & 1 \\
-1 & 0
\end{array}\right)
$$

Again in this case we can solve the equations and so we have

$$
\begin{array}{lll}
\tau(t)\left(a_{0} \otimes b_{0}\right) & = & a_{0} \otimes b_{0}, \\
\tau(t)\left(a_{1} \otimes b_{0}\right) & = & a_{0} \otimes b_{1}, \\
\tau(t)\left(a_{0} \otimes b_{1}\right) & = & -x_{1}^{-1} a_{1} \otimes b_{0}, \\
\tau(t)\left(a_{1} \otimes b_{1}\right) & = & x_{1}^{-1} a_{1} \otimes b_{1} .
\end{array}
$$

We leave the verification that $\tau(t)^{4}=1$ to the reader.

(4) $G=\mathbb{Z} / 2 \oplus \mathbb{Z} / 2$ with generators $t_{1}, t_{2}$; the only faithful indecomposable $\mathbb{Z} G^{-}$ lattice of $\mathbb{Z}$-rank 2 is given by the matrices representing $t_{1}, t_{2}$ :

$$
A_{1}=\left(\begin{array}{ll}
0 & 1 \\
1 & 0
\end{array}\right), A_{2}=\left(\begin{array}{ll}
-1 & 0 \\
0 & -1
\end{array}\right)
$$


In this case, by part (1) above and Lemma 2.4, $\tau(t)$ can be defined as follows:

$$
\begin{array}{lcc}
\tau\left(t_{2}\right)\left(a_{1} \otimes b_{0}\right) & = & -x_{1}^{-1} a_{1} \otimes b_{0}, \\
\tau\left(t_{2}\right)\left(a_{0} \otimes b_{1}\right) & = & -x_{2}^{-1} a_{0} \otimes b_{1}, \\
\tau\left(t_{1}\right)\left(a_{1} \otimes b_{0}\right) & = & a_{0} \otimes b_{1}, \\
\tau\left(t_{1}\right)\left(a_{0} \otimes b_{1}\right) & = & a_{1} \otimes b_{0}, \\
\tau\left(t_{2}\right)\left(a_{1} \otimes b_{1}\right) & = & x_{1}^{-1} x_{2}^{-1} a_{1} \otimes b_{1}, \\
\tau\left(t_{1}\right)\left(a_{1} \otimes b_{1}\right)= & -a_{1} \otimes b_{1} .
\end{array}
$$

By Lemma 2.5 it suffices to prove that $\tau\left(t_{1}\right) \tau\left(t_{2}\right)=\tau\left(t_{2}\right) \tau\left(t_{1}\right)$ :

$$
\begin{gathered}
\tau\left(t_{1}\right) \tau\left(t_{2}\right)\left(a_{1} \otimes b_{0}\right)=\tau\left(t_{1}\right)\left(-x_{1}^{-1} a_{1} \otimes b_{0}\right)=-x_{2}^{-1} a_{0} \otimes b_{1}, \\
\tau\left(t_{2}\right) \tau\left(t_{1}\right)\left(a_{1} \otimes b_{0}\right)=\tau\left(t_{2}\right)\left(a_{0} \otimes b_{1}\right)=-x_{2}^{-1} a_{0} \otimes b_{1} .
\end{gathered}
$$

Similarly we see that $\tau\left(t_{1}\right) \tau\left(t_{2}\right)\left(a_{0} \otimes b_{1}\right)=\tau\left(t_{2}\right) \tau\left(t_{1}\right)\left(a_{0} \otimes b_{1}\right)$. Next we check

$$
\begin{gathered}
\tau\left(t_{1}\right) \tau\left(t_{2}\right)\left(a_{1} \otimes b_{1}\right)=\tau\left(t_{1}\right)\left(x_{1}^{-1} x_{2}^{-1} a_{1} \otimes b_{1}\right)=-x_{1}^{-1} x_{2}^{-1} a_{1} \otimes b_{1}, \\
\tau\left(t_{2}\right) \tau\left(t_{1}\right)\left(a_{1} \otimes b_{1}\right)=\tau\left(t_{2}\right)\left(-a_{1} \otimes b_{1}\right)=-x_{1}^{-1} x_{2}^{-1} a_{1} \otimes b_{1} .
\end{gathered}
$$

(5) Dihedral group case $G=\left\langle t_{1}, t_{2} \mid t_{1}^{4}=t_{2}^{2}=e, t_{2} t_{1} t_{2}=t_{1}^{-1}\right\rangle$; the only faithful indecomposable $\mathbb{Z} G$-lattices of $\mathbb{Z}$-rank 2 are given by the matrices representing $t_{1}, t_{2}$ :

$$
\begin{array}{cl}
A_{1}=\left(\begin{array}{ll}
0 & 1 \\
-1 & 0
\end{array}\right), & A_{2}=\left(\begin{array}{ll}
1 & 0 \\
0 & -1
\end{array}\right), \\
B_{1}=\left(\begin{array}{ll}
0 & 1 \\
-1 & 0
\end{array}\right), B_{2}=\left(\begin{array}{ll}
0 & 1 \\
1 & 0
\end{array}\right) .
\end{array}
$$

In the first case we solve the equations to obtain

$$
\begin{aligned}
& \tau\left(t_{1}\right)\left(a_{1} \otimes b_{0}\right)=a_{0} \otimes b_{1}, \\
& \tau\left(t_{1}\right)\left(a_{0} \otimes b_{1}\right)=-x_{1}^{-1} a_{1} \otimes b_{0}, \\
& \tau\left(t_{2}\right)\left(a_{1} \otimes b_{0}\right)=a_{1} \otimes b_{0}, \\
& \tau\left(t_{2}\right)\left(a_{0} \otimes b_{1}\right)=-x_{2}^{-1} a_{0} \otimes b_{1}, \\
& \tau\left(t_{1}\right)\left(a_{1} \otimes b_{1}\right)=x_{1}^{-1} a_{1} \otimes b_{1}, \\
& \tau\left(t_{2}\right)\left(a_{1} \otimes b_{1}\right)=-x_{2}^{-1} a_{1} \otimes b_{1} .
\end{aligned}
$$

In the second case we have

$$
\begin{array}{llc}
\tau\left(t_{1}\right)\left(a_{1} \otimes b_{0}\right) & = & a_{0} \otimes b_{1}, \\
\tau\left(t_{1}\right)\left(a_{0} \otimes b_{1}\right) & = & -x_{1}^{-1} a_{1} \otimes b_{0}, \\
\tau\left(t_{2}\right)\left(a_{1} \otimes b_{0}\right) & = & a_{0} \otimes b_{1}, \\
\tau\left(t_{2}\right)\left(a_{0} \otimes b_{1}\right) & = & a_{1} \otimes b_{0}, \\
\tau\left(t_{1}\right)\left(a_{1} \otimes b_{1}\right) & = & x_{1}^{-1} a_{1} \otimes b_{1}, \\
\tau\left(t_{2}\right)\left(a_{1} \otimes b_{1}\right) & = & -a_{1} \otimes b_{1} .
\end{array}
$$

We will use restriction to the $p$-Sylow subgroups to obtain a cohomology calculation.

Proposition 3.2. Let $\Gamma=M \rtimes G$ denote an extension of a finite group by a finitely generated free abelian group. If the Lyndon-Hochschild-Serre spectral sequence restricted to every $p$-Sylow subgroup $G_{p} \subset G$ collapses at $E_{2}$, without extension problems, then the same is true for the LHS spectral sequence of the original extension. 
Proof. The restriction induces an embedding $H^{i}\left(G, H^{j}(M)\right)_{(p)} \subset H^{i}\left(G_{p}, H^{j}(M)\right)$ for all $i>0$, whereas $H^{j}(M)^{G} \subset H^{j}(M)^{G_{p}}$. Hence we deduce that the differentials are trivial at $p$, for all primes $p$ dividing $|G|$. As the images always lie in torsion modules annihilated by $|G|$, we deduce that there can be no differentials. Similarly if there are no extension problems $p$-locally, they cannot occur integrally.

Corollary 3.3. If $M \rtimes G$ admits a compatible action for all $p$-Sylow subgroups $G_{p} \subset G$, then the LHS spectral sequence for the extension collapses at $E_{2}$ without extension problems.

We can now prove our main result.

Theorem 3.4. If $M$ is a $\mathbb{Z} G$-lattice which restricts on each p-Sylow subgroup of $G$ to a direct sum of finitely many one- and two-dimensional integral representations, then for all integers $k \geq 0$ we have

$$
H^{k}(M \rtimes G, \mathbb{Z}) \cong \bigoplus_{i+j=k} H^{i}\left(G, H^{j}(M, \mathbb{Z})\right) .
$$

Proof. We need only consider $p$-groups and integral representations of rank one or two which are faithful. It is clear that $G L_{1}(\mathbb{Z}) \cong \mathbb{Z} / 2$, whereas the complete list of finite $p$-subgroups of $G L_{2}(\mathbb{Z})$ is given by

- The cyclic subgroups $\mathbb{Z} / 2, \mathbb{Z} / 3, \mathbb{Z} / 4$.

- $\mathbb{Z} / 2 \times \mathbb{Z} / 2$.

- The dihedral group $D_{8}$ of order eight.

We have constructed compatible actions for all of the faithful integral representations of these groups having rank one or two. Hence by Corollary 3.3, we obtain the desired decomposition theorem.

\section{TOROIDAL ORBIFOLDS}

In this section we will outline how integral representations of finite groups naturally give rise to group actions on tori. The resulting orbifolds appear as examples of interest in physics, and we will provide explicit computations in the next section.

Assume that $G$ is a finite group and that we are given a faithful integral representation $G \subset G L_{n}(\mathbb{Z})$. This will induce a smooth action of $G$ on $\mathbb{R}^{n}$ via matrices. Given that these matrices have integer entries, this will induce an action on the quotient $X=\mathbb{R}^{n} / \mathbb{Z}^{n}$, where $\mathbb{Z}^{n} \subset \mathbb{R}^{n}$ is the canonical lattice of rank $n$. Note that the action will always have a fixed point (indeed the zero vector is invariant). The space $Y=X / G$ is what is often denoted as a global quotient, and $X \rightarrow X / G$ carries a natural orbifold structure.

Let $E G$ denote a contractible free $G$-space, and consider the Borel construction $E G \times_{G} X$. Projection on the first coordinate yields a fibration $X \rightarrow E G \times_{G} X \rightarrow$ $B G$, where $B G=E G / G$ is the classifying space of $G$. Note that both $X$ and $B G$ have contractible universal covers, hence $E G \times_{G} X$ is aspherical. This space can be regarded as the classifying space of the orbifold, and $\Gamma=\pi_{1}\left(E G \times_{G} X\right)$ is known as the orbifold fundamental group. Note that the cohomology of this group is by definition the cohomology of its classifying space, which in this case is precisely $E G \times{ }_{G} X$.

The group $\Gamma$ fits into a group extension of the form $1 \rightarrow M \rightarrow \Gamma \rightarrow G \rightarrow 1$, where $M \cong \mathbb{Z}^{n}$ has the $G$ action induced from the representation $G \subset G L_{n}(\mathbb{Z})$, i.e., $M$ is a $\mathbb{Z}[G]$ lattice. This extension arises from applying $\pi_{1}$ to the fibration above. 
Note that the projection $E G \times_{G} X \rightarrow B G$ has a section, due to the fact that the $G$-action has a fixed point. Hence the extension splits, and so $\Gamma \cong M \rtimes G$, the semidirect product determined by the action of $G$ on $M$ (up to isomorphism). We will be interested in knowing how many different splittings this extension has. This is measured by the cohomology group $H^{1}(G, M)$. It can also be identified with $M$-conjugacy classes of subgroups $\tilde{G} \subset \Gamma$ mapping isomorphically onto $G$.

We now express this information geometrically. The $G$-action on $X$ lifts to a $\Gamma$ action on $Z=\mathbb{R}^{n}$. As pointed out in [5], page 267 we have, for any $Q \subset G$,

$$
X^{Q}=\bigsqcup_{H^{1}(Q, M)} Z^{\tilde{Q}} / M \cap N(\tilde{Q}),
$$

where $N(\tilde{Q})$ is the normalizer of $\tilde{Q}$. We can also consider the coefficient sequence

$$
0 \rightarrow M \rightarrow M \otimes \mathbb{R} \rightarrow X \rightarrow 0,
$$

which gives rise to the exact sequence

$$
0 \rightarrow M^{Q} \rightarrow(M \otimes \mathbb{R})^{Q} \rightarrow X^{Q} \rightarrow H^{1}(Q, M) \rightarrow 0 .
$$

From this we see that for every $Q \subset G$, the $Q$-fixed point set in $X$ will always decompose into a disjoint union of tori. Comparing the two expressions we see that $Z^{\tilde{Q}}$ is isomorphic to $\mathbb{R}^{n_{Q}}$, where $n_{Q}$ is the rank of the free abelian group $M^{Q}$, the subgroup of $Q$-invariants, and that $M \cap N(\tilde{Q}) \cong \mathbb{Z}^{n_{Q}}$. Indeed we obtain the formula

$$
X^{Q}=\bigsqcup_{H^{1}(Q, M)}\left(\mathbb{S}^{1}\right)^{r k_{\mathbb{Z}}\left[M^{Q}\right]} .
$$

Note in particular that if $M^{Q}=\{0\}$, then $X^{Q}$ will be a disjoint collection of points indexed by $H^{1}(Q, M)$.

In differential geometry examples arise using complex coordinates. More precisely if say $G \subset G L_{m}(\mathbb{C})$ and there exists a rank $2 m$ lattice $\Lambda \subset \mathbb{C}^{m}$ such that $G \Lambda \subset \Lambda$, then this will induce a $G$-action on $\left(\mathbb{S}^{1}\right)^{2 m}=\mathbb{C}^{m} / \Lambda$. The action on the lattice $\Lambda$ (assumed to be faithful) provides an embedding $G \subset G L(\Lambda)$ from which we derive the same data as before and can therefore construct a topologically equivalent model.

\section{Key examples and CALCUlations}

In this section we will apply our techniques to certain very specific examples arising from geometry and physics. Given an orbifold $X \rightarrow X / G$, our main object of interest will be the group of gerbes associated to it. We shall not dwell here on the definition of gerbes, but use the following identification for the isomorphism classes of gerbes and flat gerbes, respectively:

$$
G b(X / G)=H^{3}\left(E G \times_{G} X, \mathbb{Z}\right), \quad F G b(X / G)=H^{2}\left(E G \times_{G} X, \mathbb{U}(1)\right) .
$$

For a finitely generated abelian group $A$, let $A=T(A) \oplus F(A)$ denote the decomposition into torsion and torsion-free components, respectively. Then, by the universal coefficient theorem, we have

$$
\begin{gathered}
G b(X / G)=T\left(H_{2}\left(E G \times_{G} X, \mathbb{Z}\right)\right) \oplus F\left(H_{3}\left(E G \times_{G} X, \mathbb{Z}\right)\right), \\
F G b(X / G)=T\left(H_{2}\left(E G \times_{G} X, \mathbb{Z}\right)\right) \oplus F\left(H_{2}\left(E G \times_{G} X, \mathbb{Z}\right)\right) \otimes U(1) .
\end{gathered}
$$

For our examples, these calculations will amount to computing the low-dimensional cohomology of certain discrete groups. In particular we will be computing 
the Schur multiplier $H^{2}\left(M \rtimes G, \mathbb{S}^{1}\right)$. This is a problem of independent interest in group cohomology, and our methods indicate a practical strategy for computing this invariant for many examples.

First we introduce some general notation. If $M$ is a $\mathbb{Z} G$-lattice building the semidirect product $M \rtimes G$, then the cohomology of the associated torus can be described as an exterior algebra $\wedge^{*}\left(M^{*}\right)$ where $M^{*}$ is the dual of $M$. The action on these exterior powers is determined by the original action on $M$. From the point of view of group cohomology we should observe that for $G$ having cyclic $p$-sylow subgroups, any $\mathbb{Z} G$-lattice will be cohomologous to its dual.

As would be expected, the most interesting examples, and the real motivation for this work, comes from calculations for six-dimensional orbifolds, where the usual spectral sequence techniques become rather complicated. Here our methods provide an important new ingredient that will allow us to compute rigorously beyond the known range. An important class of examples in physics arise from actions of a cyclic group $\mathbb{Z} / N$ on $\mathbb{T}^{6}$. In our scheme, these come from six-dimensional integral representations of $\mathbb{Z} / N$. However, the constraints from physics impose certain restrictions on them (see [6]). If $\theta \in G L_{6}(\mathbb{Z})$ is an element of order $N$, then it can be diagonalized over the complex numbers. The associated eigenvalues, denoted $\alpha_{1}, \alpha_{2}, \alpha_{3}$, should satisfy $\alpha_{1} \alpha_{2} \alpha_{3}=1$, and in addition all of the $\alpha_{i} \neq 1$. The first condition implies that the orbifold $\mathbb{T}^{6} \rightarrow \mathbb{T}^{6} / G$ is a Calabi-Yau orbifold, and so admits a crepant resolution. These more restricted representations have been classified in $[6$, where it is shown that there are precisely 18 inequivalent lattices of this type. Six of them decompose as direct sums of sublattices of rank two or less, and so we can apply Theorem 3.4 to obtain a computation of the cohomology of the corresponding discrete groups. It would seem likely that an extension of our methods should also apply to the other examples on this list. We will provide a complete computation for the following celebrated example, which appears in [1].

5.1. The case $Y_{1}=T^{6} / \mathbb{Z} / 4$. The action of $\mathbb{Z} / 4$ on $T^{6}$ is induced from the action of $\mathbb{Z} / 4$ on $\mathbb{C}^{3}$, given by

$$
\kappa\left(z_{1}, z_{2}, z_{3}\right)=\left(-z_{1}, i z_{2}, i z_{3}\right) .
$$

The induced action of $\mathbb{Z} / 4$ on $\mathbb{Z}^{6}$ is given by the following decomposition of $\mathbb{Z}[\mathbb{Z} / 4]$ lattices:

$$
M=\left(M_{1}\right)^{2} \oplus\left(M_{2}\right)^{2}
$$

where $M_{1}$ has rank one and $\kappa$ acts by multiplication by -1 , and $M_{2}$ has rank two and $\kappa$ acts by the following rule $\kappa(x)=-y, \kappa(y)=x$, i.e., the standard embedding $\mathbb{Z} / 4 \subset G L_{2}(\mathbb{Z})$. We observe that this module is a sum of lattices for which our methods apply, hence computing the cohomology of the associated semi-direct product reduces to determining the $E_{2}$ term of the LHS spectral sequence.

First note that $M \cong M^{*}$, so what we need to do is determine the exterior powers of $M$. Note that $\bigwedge^{i}(M) \cong \bigwedge^{6-i}(M)^{*} \cong \bigwedge^{6-i}(M)$. Hence we only need to worry about $\Lambda^{2}$ and $\Lambda^{3}$. We will use the distributive formula for exterior powers of a direct sum. Now it is easy to see that $M_{1} \otimes M_{1} \cong \mathbb{Z}$, the trivial module, whereas $M_{1} \otimes M_{2} \cong M_{2}$. For the tensor product $M_{2} \otimes M_{2}$ we have a basis with action given by $x \otimes x \rightarrow y \otimes y, x \otimes y \mapsto-y \otimes x, y \otimes x \mapsto-x \otimes y$ and $y \otimes y \mapsto x \otimes x$.

\footnotetext{
${ }^{4}$ In the language of physics, they show that there exist 18 inequivalent $N=1$ supersymmetric string theories on symmetric orbifolds of $(2,2)$-type without discrete background.
} 
Hence $M_{2} \otimes M_{2} \cong P \oplus P$, where $P \cong \mathbb{Z}[\mathbb{Z} / 4] \otimes_{\mathbb{Z} / 2} \mathbb{Z}$ is the inflation of the regular representation of $\mathbb{Z} / 2$.

Now we may compute

$$
\bigwedge^{2}(M) \cong(\mathbb{Z})^{3} \oplus\left(M_{2}\right)^{4} \oplus(P \oplus P), \quad \bigwedge^{3}(M) \cong\left(M_{1}\right)^{4} \oplus\left(M_{2}\right)^{4} \oplus(P)^{4} .
$$

Summarizing, we have

$$
H^{i}\left(\mathbb{Z}^{6}, \mathbb{Z}\right)= \begin{cases}\mathbb{Z} & \text { if } i=0,6, \\ M_{1}^{2} \oplus M_{2}^{2} & \text { if } i=1,5, \\ \mathbb{Z}^{3} \oplus\left(M_{2}\right)^{4} \oplus(P)^{2} & \text { if } i=2,4, \\ \left(M_{1}\right)^{4} \oplus\left(M_{2}\right)^{4} \oplus(P)^{4} & \text { if } i=3, \\ 0 & \text { if } i>6 .\end{cases}
$$

Next we compute the terms $H^{s}\left(\mathbb{Z} / 4, H^{t}\left(\mathbb{Z}^{6}, \mathbb{Z}\right)\right)$. These can be reduced to computing the cohomology of $\mathbb{Z} / 4$ with coefficients in the modules $\mathbb{Z}, M_{1}, M_{2}$ and $P$. By an elementary calculation we have that

$$
\begin{aligned}
H^{i}(\mathbb{Z} / 4, \mathbb{Z})= \begin{cases}\mathbb{Z} & \text { if } i=0, \\
0 & \text { if } i \text { is odd, } \\
\mathbb{Z} / 4 & \text { if } i>0 \text { is even, }\end{cases} \\
H^{i}\left(\mathbb{Z} / 4, M_{1}\right)= \begin{cases}0 & \text { if } i=0, \\
\mathbb{Z} / 2 & \text { if } i \text { is odd }, \\
0 & \text { if } i>0 \text { is even, }\end{cases} \\
H^{i}\left(\mathbb{Z} / 4, M_{2}\right)= \begin{cases}0 & \text { if } i=0, \\
\mathbb{Z} / 2 & \text { if } i \text { is odd }, \\
0 & \text { if } i>0 \text { is even, }\end{cases} \\
H^{i}(\mathbb{Z} / 4, P)= \begin{cases}\mathbb{Z} & \text { if } i=0, \\
0 & \text { if } i \text { is odd }, \\
\mathbb{Z} / 2 & \text { if } i>0 \text { is even. }\end{cases}
\end{aligned}
$$

From this the cohomology can easily be written down as

$$
H^{i}\left(\mathbb{Z}^{6} \rtimes \mathbb{Z} / 4, \mathbb{Z}\right)= \begin{cases}\mathbb{Z} & \text { if } i=0, \\ 0 & \text { if } i=1, \\ \mathbb{Z}^{5} \oplus \mathbb{Z} / 4 \oplus(\mathbb{Z} / 2)^{4} & \text { if } i=2, \\ \mathbb{Z}^{4} \oplus(\mathbb{Z} / 2)^{4} & \text { if } i=3, \\ \mathbb{Z}^{5} \oplus(\mathbb{Z} / 4)^{4} \oplus(\mathbb{Z} / 2)^{14} & \text { if } i=4, \\ (\mathbb{Z} / 2)^{12} & \text { if } i=5, \\ \mathbb{Z} \oplus(\mathbb{Z} / 4)^{7} \oplus(\mathbb{Z} / 2)^{20} & \text { if } i=6, \\ (\mathbb{Z} / 2)^{12} & \text { if } i=2 k+1, k>1, \\ (\mathbb{Z} / 4)^{8} \oplus(\mathbb{Z} / 2)^{20} & \text { if } i=2 k, k>3 .\end{cases}
$$

We obtain a calculation for the group of gerbes:

$$
F G b\left(T^{6} / \mathbb{Z} / 4\right)=U(1)^{5} \oplus(\mathbb{Z} / 2)^{4}, G b\left(T^{6} / \mathbb{Z} / 4\right)=\mathbb{Z}^{4} \oplus(\mathbb{Z} / 2)^{4} .
$$

We now offer an interpretation of this calculation. The fact that $H^{1}(\mathbb{Z} / 2, M) \cong$ $(\mathbb{Z} / 2)^{4}$ means that there are sixteen distinct $M$-equivalence classes of subgroups of order two in $\Gamma=M \rtimes \mathbb{Z} / 4$. Of these sixteen classes, twelve of them have a normalizer of the form $\mathbb{Z}^{2} \times \mathbb{Z} / 2$, and four of them have normalizer $\mathbb{Z}^{2} \rtimes \mathbb{Z} / 4$, where $\mathbb{Z} / 4$ acts via multiplication by $-I$ on $\mathbb{Z}^{2}$. In $\Gamma$, the twelve conjugacy classes are identified in pairs, and so we obtain the following proposition. 
Proposition 5.1. The group $\Gamma=M \rtimes \mathbb{Z} / 4$ has ten conjugacy classes of subgroups of order two. Six of them have normalizers $\mathbb{Z}^{2} \times \mathbb{Z} / 2$ and four of them have normalizers $\mathbb{Z}^{2} \rtimes \mathbb{Z} / 4$.

Given that $\Gamma$ is a group of finite virtual cohomological dimension with periodic cohomology, we can apply a formula due to Brown (see [5], page 293) for the highdimensional cohomology of $\Gamma$, expressible as the sum of the cohomology of the normalizers of elements of order two, which in this case becomes (for $i>6$ )

$$
H^{i}(\Gamma, \mathbb{Z}) \cong\left[H^{i}\left(\mathbb{Z}^{2} \times \mathbb{Z} / 2, \mathbb{Z}\right)\right]^{6} \oplus\left[H^{i}\left(\mathbb{Z}^{2} \rtimes \mathbb{Z} / 4, \mathbb{Z}\right)\right]^{4},
$$

which indeed coincides with our computation.

D. Joyce (8], page 861) has constructed five different desingularizations of the orbifold $Y_{1}$. His construction arises from desingularizing the orbifolds $\mathbb{T}^{2} /\{ \pm I\}$ associated to the four centralizers of the form $\mathbb{Z}^{2} \rtimes \mathbb{Z} / 4$. Each of them can be resolved in two possible ways, and this leads to the existence of five different possibilities. From the point of view of group cohomology, we see that these centralizers are captured by the high-dimensional cohomology. In fact the Farrell-Tate Cohomology of the orbifold fundamental group seems to be a natural repository for the relevant cohomological data. For our example this invariant is periodic of period two, and equals $\widehat{H}^{1}(\Gamma) \cong(\mathbb{Z} / 2)^{12}, \widehat{H}^{2}(\Gamma) \cong(\mathbb{Z} / 4)^{8} \oplus(\mathbb{Z} / 2)^{20}$. It would seem from this calculation that it is naive to expect that desingularizations can be read off from the cohomology. On the other hand we can see that the cohomology does contain important information about the singular set.

5.2. The case $Y_{2}=T^{6} /(\mathbb{Z} / 2)^{2}$. Here the action of $(\mathbb{Z} / 2)^{2}$ on $T^{6}$ is also induced from the action of $\mathbb{Z} / 2^{2}$ on $\mathbb{C}^{3}$, given on generators by

$$
\sigma_{1}\left(z_{1}, z_{2}, z_{3}\right)=\left(-z_{1},-z_{2}, z_{3}\right), \quad \sigma_{2}\left(z_{1}, z_{2}, z_{3}\right)=\left(-z_{1}, z_{2},-z_{3}\right) .
$$

The induced action of $\mathbb{Z} / 2^{2}$ on $\mathbb{Z}^{6}$ is given by the following decomposition of $(\mathbb{Z} / 2)^{2}$ modules:

$$
M=\left(L_{1}\right)^{2} \oplus\left(L_{2}\right)^{2} \oplus\left(L_{3}\right)^{2},
$$

where $L_{1}$ has rank one and $\sigma_{i}, i=1,2$, act by multiplication by $-1 ; L_{2}$ has rank one, $\sigma_{1}$ acts by multiplication by $-1, \sigma_{2}$ acts by multiplication by 1 ; and $L_{3}$ also has rank one and $\sigma_{1}$ acts by multiplication by 1 and $\sigma_{2}$ acts by multiplication by -1 .

As before, we need a decomposition of the exterior powers of $M$; however as it breaks up into one-dimensional summands this is easy to obtain. Note that $L_{i} \otimes L_{i} \cong \mathbb{Z}$, whereas $L_{i} \otimes L_{j} \cong L_{k}$, where $\{i, j, k\}=\{1,2,3\}$ :

$$
H^{i}\left(\mathbb{Z}^{6}, \mathbb{Z}\right)= \begin{cases}\mathbb{Z} & \text { if } i=0,6, \\ L_{1}^{2} \oplus L_{2}^{2} \oplus L_{3}^{2} & \text { if } i=1,5, \\ \mathbb{Z}^{3} \oplus L_{1}^{4} \oplus L_{2}^{4} \oplus L_{3}^{4} & \text { if } i=2,4, \\ \mathbb{Z}^{8} \oplus L_{1}^{4} \oplus L_{2}^{4} \oplus L_{3}^{4} & \text { if } i=3, \\ 0 & \text { if } i>6 .\end{cases}
$$

We now compute the first few cohomology groups for $\mathbb{Z}^{6} \rtimes(\mathbb{Z} / 2)^{2}$. First we observe that if $G=(\mathbb{Z} / 2)^{2}$, then for each $i=1,2,3$ there exists an index two subgroup $H_{i} \subset G$ such that $L_{i}$ fits into a short exact sequence $0 \rightarrow L_{i} \rightarrow \mathbb{Z}\left[G / H_{i}\right] \rightarrow \mathbb{Z} \rightarrow 0$. From this we conclude that $H^{1}\left(G, L_{i}\right) \cong \mathbb{Z} / 2$ and $H^{2}\left(G, L_{i}\right) \cong \mathbb{Z} / 2$. Hence we have 
that

$$
H^{i}\left(\mathbb{Z}^{6} \rtimes \mathbb{Z} / 2^{2}, \mathbb{Z}\right)= \begin{cases}\mathbb{Z} & \text { if } i=0, \\ 0 & \text { if } i=1, \\ \mathbb{Z}^{3} \oplus(\mathbb{Z} / 2)^{6} & \text { if } i=2, \\ \mathbb{Z}^{8} \oplus(\mathbb{Z} / 2)^{18} & \text { if } i=3 .\end{cases}
$$

From this we derive the desired information about gerbes:

$$
F G b\left(T^{6} /(\mathbb{Z} / 2)^{2}\right)=U(1)^{3} \oplus(\mathbb{Z} / 2)^{18}, G b\left(T^{6} /(\mathbb{Z} / 2)^{2}\right)=\mathbb{Z}^{8} \oplus(\mathbb{Z} / 2)^{18} .
$$

Of course we could write down the entire cohomology with integral coefficients, but that would not be particularly illuminating. However we can use $\mathbb{F}_{2}$ coefficients instead; the spectral sequence still collapses, and the complete ring structure is given up to filtration by

$$
H^{*}\left(\mathbb{Z}^{6} \rtimes(\mathbb{Z} / 2)^{2}, \mathbb{F}_{2}\right) \cong \mathbb{F}_{2}[u, v] \otimes \bigwedge\left(x_{1}, x_{2}, x_{3}, x_{4}, x_{5}, x_{6}\right),
$$

where the generators are all in degree one. Note that the fixed point set of this action consists of $2^{6}=64$ isolated points, and that the total dimension of the cohomology of the torus is also $2^{6}$. This is of course consistent with the collapse of the spectral sequence and the localization theorem.

This can be stated as a special case of a general theorem:

Theorem 5.2. Suppose that $G$ is a finite group and we are given a homomorphism

$$
G \rightarrow G L_{1}(\mathbb{Z})^{n} \subset G L_{n}(\mathbb{Z}) .
$$

If $\Gamma=M \rtimes G$ where $M$ is the resulting $\mathbb{Z}[G]$ lattice, then

$$
H^{*}\left(\Gamma, \mathbb{F}_{2}\right) \cong H^{*}\left(G, \mathbb{F}_{2}\right) \otimes \bigwedge^{*}(M) .
$$

From this example we see that the cohomology will grow very rapidly, as is to be expected in a non-periodic situation. This is reflected in the large number of desingularizations which have been described in 8]. Again we see that the cohomological computation provides insight as to the complicated possibilities for desingularization, but their precise determination is quite complex.

\section{REFERENCES}

[1] Adem, A., $\mathbb{Z} / p \mathbb{Z}$ actions on $\left(\mathbb{S}^{n}\right)^{k}$, Trans. Amer. Math. Soc. 300 (1987), no. 2, 791-809. MR0876479 (88b:57037)

[2] Adem, A. and Milgram, R.J., Cohomology of finite groups, Grundlehren der Mathematischen Wissenschaften 309, Springer-Verlag, Berlin, 1994. MR.1317096 (96f:20082)

[3] de Boer, J., Dijkgraaf, R., Hori, K., Keurentjes, A., Morgan, J., Morrison, D. and Sethi, S., Triples, Fluxes, and Strings, Adv. Theor. Math. Phys. 4 (2000), no. 5, 995-1186. MR.1868756 (2002i:81186)

[4] Brady, T., Free resolutions for semi-direct products, Tohoku Math. J. (2) 45 (1993), no. 4, 535-537. MR1245720 (94f:20096)

[5] Brown, K., Cohomology of groups, Grad. Texts in Mathematics 87 Springer-Verlag, New York, 1982. MR0672956 (83k:20002)

[6] Erler, J. and Klemm, A., Comment on the generation number in orbifold compactifications, Comm. Math. Phys. 153 (1993), 579-604. MR1218933 (94i:32044)

[7] Evens, L., Cohomology of groups, Oxford Mathematical Monographs, Oxford University Press (1991). MR1144017 (93i:20059)

[8] Joyce, D., Deforming Calabi-Yau orbifolds, Asian J. Math. 3 (1999), no. 4, 853-867. MR:1797581 (2001i:14004)

[9] Lupercio, E. and Uribe, B., Gerbes over orbifolds and twisted K-theory, Comm. Math. Phys. 245 (2004), 449-489. MR2045679 (2005m:53035) 
[10] Ruan, Y., Discrete torsion and twisted orbifold cohomology, J. Symplectic Geometry 2 (2003), 1-24. MR2128387

[11] Vafa, C. and Witten, E., On orbifolds with discrete torsion, J. Geom. Phys. 15 (1995), no. 3, 189-214. MR $1316330(95 \mathrm{~m}: 81190)$

[12] Vafa, C., Modular invariance and discrete torsion on orbifolds, Nuclear Phys. B 273 (1986), no. 3-4, 592-606. MR0850976 (87j:81232)

Department of Mathematics, University of Wisconsin, Madison, Wisconsin 53706

E-mail address: adem@math.wisc.edu

Current address: Department of Mathematics, University of British Columbia, Vancouver, British Columbia, Canada V6T 1Z2

E-mail address: adem@math.ubc.ca

Institute of Mathematics, Academia Sinica, Beijing 100080, People's Republic of CHINA

E-mail address: pjz@math03.math.ac.cn 